\title{
Can we still consider thyroid hyperfunction a protective condition for the onset of thyroid cancer?
}

\author{
Elena Bonati, Stefania Bettoni, Tommaso Loderer, Paolo Del Rio \\ General Surgery Unit, Department of Medicine and Surgery, Parma University Hospital, Parma, Italy \\ Contributions: (I) Conception and design: E Bonati, P Del Rio; (II) Administrative support: E Bonati, S Bettoni; (III) Provision of study materials or \\ patients: E Bonati, P Del Rio, S Bettoni; (IV) Collection and assembly of data: T Loderer, E Bonati; (V) Data analysis and interpretation: E Bonati, \\ T Loderer, S Bettoni; (VI) Manuscript writing: All authors; (VII) Final approval of manuscript: All authors. \\ Correspondence to: Elena Bonati. General Surgery Unit, Department of Medicine and Surgery, Parma University Hospital, 14 Gramsci Road, Parma, \\ Italy. Email: ebonati86@gmail.com; elena.bonati@unipr.it.
}

Background: Thyroid cancer is the ninth most commonly diagnosed cancer in the world, and the most common endocrine carcinoma. It was originally believed to be a rare event in patients with thyroid hyperfunction and it was reported that hyperthyroidism had a protective role against thyroid neoplasms. However, in recent years, several studies have hypothesized that differentiated thyroid carcinomas and hyperthyroidism may coexist. Our study aims therefore to evaluate the incidence of differentiated thyroid carcinomas on definitive histological examination, in patients undergoing total thyroidectomy or hemithyroidectomy with coexisting hyperfunctioning thyroid disease, to understand whether hyperthyroidism can be considered a protective condition against the onset of thyroid neoplasms.

Methods: The study involved 1,449 patients underwent to thyroid surgery from 2010 to 2018 at the General Surgery Unit, Department of Surgery, University Hospital of Parma, Parma, Italy, presenting thyroid cancer at postoperative histological exam. Patients were divided in two groups based on the presence (Group A) or absence (Group B) of hyperfunction. All data were collected in a dedicated database and include demographic data, such as age and sex, preoperative cytology, date and type of surgery, postoperative diagnosis, characteristics of aggressiveness of the neoplasm and postoperative complications. For data analysis, a $\mathrm{P}$ value of less than 0.05 was considered statistically significant.

Results: The incidence of thyroid carcinomas was lower in patients suffering from hyperfunction compared to the incidence found in non-hyperthyroid patients, both in preoperative cytological examination and in postoperative diagnosis through histological examination. Furthermore, the tumors that have developed in patients with hyperfunction had a comparable degree of aggression and invasiveness in the two groups studied. However, we have found an equal incidence of microcarcinomas and occult carcinomas on postoperative histological examination. Postoperative complications in patients with cancer were similar, regardless of the presence or absence of hyperfunctioning thyroid disease.

Conclusions: Our study confirms that hyperthyroidism is a protective condition against thyroid carcinoma, but the finding of an equivalent incidence of occult carcinomas in the two groups stresses the need to perform a cytological examination in case of a nodular pathology in a hyperthyroid patient before performing a treatment.

Keywords: Thyroid; hyperthyroidism; thyroid cancer; occult carcinoma; microcarcinoma

Submitted Aug 28, 2020. Accepted for publication Dec 18, 2020.

doi: $10.21037 /$ gs-20-688

View this article at: http://dx.doi.org/10.21037/gs-20-688 


\section{Introduction}

Thyroid cancer represents a significant health problem spread across the world. It is the ninth malignancy by incidence and have a mortality rate of 0.5 per 100,000 (1). It is the most frequent malignant endocrine neoplasms, over $50 \%$ of total cases are diagnosed before the age of 55 , with a higher prevalence in women. The incidence of occult and asymptomatic neoplasms in recent years has been increasing, due to the greater sensitivity towards thyroid disease, the introduction of increasingly expanded screening programs, the use of more precise ultrasound diagnostic techniques.

Originally, thyroid cancer was considered a rare phenomenon among patients affected by thyroid hyperfunction (2), and Basedow-Graves was even described as an "insurance against thyroid cancer" (3). However, many recent studies have reported an increased prevalence of differentiated thyroid carcinoma in hyperthyroid patients compared to the healthy population. Current hypotheses regarding the mechanism of carcinogenesis focus on the signal transmission pathways activated by the binding between thyroid-stimulating autoantibodies (4). In fact, it is thought that the activated signaling pathways could equally favor growth, invasion, neoangiogenesis and activate the IGF-1 (insulin-like growth factor) pathway (2). For these reasons, the coexistence of thyroid carcinoma and hyperthyroidism, in particular of Basedow-Graves' disease must no longer be considered rare (5).

The increase in prevalence could be a result of the increased use of thyroidectomy as a treatment of choice for persistent hyperthyroidism, as it increases the probability of diagnosing occult carcinomas, which have a prevalence between $0.45 \%$ and $35.6 \%$ (6). In addition, occult thyroid lesions can be revealed through ultrasound follow-up of hyperthyroid disease. Thyroid chronic stimulation by thyroid stimulating autoantibodies (TRAb) could facilitate the neoplastic transformation of thyrocytes in genetically predisposed individuals. In particular, the genetic mutations concern specific proto-oncogenic drugs, such as the RET or TRK gene, which would lead to the development of papillary carcinoma (7). In addition, some dietary factors may also be relevant. In fact, in most cases, papillary carcinoma is found in hyperthyroid patients and it is notoriously associated with iodine intake (8).

The aim of the study was to evaluate the incidence of differentiated thyroid cancer, on definitive histological examination, in patients undergoing total thyroidectomy or hemithyroidectomy for hyperfunctioning thyroid disease, to understand whether hyperthyroidism can be considered a protective condition against the onset of thyroid cancer. In particular, the aforementioned incidence will be estimated both in patients who have carried out a preoperative cytological examination, and in patients who have not carried out the cytological examination because they do not suffer from a nodular pathology. In addition, a further objective will be the comparative assessment of the aggressiveness and invasiveness of the carcinomas found in patients with hyperthyroidism and non-hyperthyroid patients, to verify whether thyroid carcinomas manifest themselves with a variable or similar aggressiveness in the two different groups studied. Finally, the frequency of postoperative complications recorded in these patients, such as dysphonia, dysphagia, dyspnea, paresthesia and bleeding, will be evaluated.

We present the following article in accordance with the STROBE reporting checklist (available at http://dx.doi. org/10.21037/gs-20-688).

\section{Methods}

The retrospective observational study involved all patients who underwent total thyroidectomy or hemithyroidectomy from January 2010 to December 2018 at the General Surgery Unit, Department of Surgery, University Hospital of Parma, Parma, Italy, including patients not suffering from hyperfunctioning thyroid disease (Group B) and hyperthyroid patients suffering from the disease BasedowGraves', multinodular toxic goiter and toxic Plummer's adenoma (Group A).

It is important to specify that the study is conducted in an iodine-deficient region, endemic for goiter, in which the population therefore has a tendency to hypothyroidism rather than hyperfunction.

Data were collected in a dedicated and authorized by an institutional review board database and includes demographic data, such as gender and age; presence of thyroid hyperfunction; preoperative cytology, according to the Bethesda classification (Thyr 1-6); date of the intervention, from 2010 to 2018; type of surgery performed (total thyroidectomy or hemithyroidectomy); definitive postoperative histological examination; presence of anatomopathological features related to the aggressiveness of the neoplasm, such as sclerosis, oxyphilia, high cells, perithyroid tissue infiltration, multifocality and lymph node metastases; any postoperative complications found 
Table 1 Demographic patients characteristics

\begin{tabular}{|c|c|c|c|c|c|c|}
\hline Characteristics & \multicolumn{2}{|c|}{ Total } & \multicolumn{2}{|c|}{ Group A } & \multicolumn{2}{|c|}{ Group B } \\
\hline Age (mean) & 54.04 & & 55.05 & & 53.71 & \\
\hline \multicolumn{7}{|l|}{ Sex } \\
\hline Female & 1,154 & 79.6 & 291 & 83.4 & 863 & 78.5 \\
\hline \multicolumn{7}{|l|}{ Intervention } \\
\hline Lobectomy & 254 & 17.5 & 41 & 11.7 & 213 & 19.4 \\
\hline Thyroidectomy & 1,195 & 82.5 & 308 & 88.3 & 887 & 80.6 \\
\hline
\end{tabular}

in patients with carcinoma, such as dysphagia, dysphonia, dyspnea, paresthesia and bleeding.

\section{Statistical analysis}

All patients with incomplete data collection were excluded from the study. Data were analyzed using a chi-square test and Fisher's exact test, to compare parametric variables, while Mann-Whitney's U-test was used to compare nonparametric variables. $\mathrm{P}$ values $<0.05$ were defined as statistically significant.

\section{Ethical statement}

The study was conducted in accordance with the Declaration of Helsinki (as revised in 2013). The study was approved by institutional ethics board of Parma - AVEN (No. 1009/2019/OSS*/AOUPR) and individual consent for this retrospective analysis was waived.

\section{Results}

A total of 1,449 patients, eligible and with complete data, were enrolled in our study (1,154 females and 295 males; with a mean age of $54.04 \pm 13.71$ ). A personal history of hyperthyroidism was reported, and confirmed at histological examination, in 349 patients $(24.1 \%$, Group A) while 1,100 had no hyperfunction thyroid disease (Group B). Hyperfunction had a frequency of $25.2 \%$ in female patients, with 291 cases total, while in males, hyperfunction has a frequency of $19.7 \%$, with 58 cases $(\mathrm{P}<0.05)$. Group A patients had a mean age of $55.05 \pm 14.12$ while Group B had $53.71 \pm 13.573(\mathrm{P}>0.05)$.
Overall, patients undergoing total thyroidectomy were $1,195(82.5 \%)$ while those undergoing hemithyroidectomy were 254 (17.5\%). In Group A, 308 patients underwent total thyroidectomy, representing $88.3 \%$, while the remaining 41 patients, as well as $11.7 \%$, underwent hemithyroidectomy. In Group B, total thyroidectomy was performed in $80.6 \%$ (887 patients) and hemithyroidectomy in $19.4 \%$ (213 patients) (Table 1).

Fine needle aspiration was preoperatively performed in 1,169 patients and the exam was reported according to 2017 Bethesda System for Reporting Thyroid Cytopathology (Table 2) (9).

Among patients with hyperfunction, a preoperative diagnosis of benignity (category Thyr 2) prevails. In addition, classes associated to certain or suspected malignancy have lower frequencies in hyperfunctioning patients compared to patients who do not have hyperfunction $(\mathrm{P}<0.05)$.

A total of 280 patients did not carry out preoperative cytological examination, and 168 of them presented thyroid hyperfunction. Hyperfunctioning patients in whom the examination was not performed represent $48.1 \%$ of all patients in Group A, while among Group B only $10.2 \%$ did not perform preoperative needle aspiration. This substantial difference is attributable to a reduced incidence of nodular pathology in hyperthyroid patients, who in most cases have diffuse glandular pathology and therefore often do not need to carry out a cytological examination.

Subsequently, the incidence of carcinomas and microcarcinomas was assessed at the postoperative diagnosis, through a definitive histological examination, both in patients with hyperfunction and in patients who do not have hyperfunction. Out of 1,444 patients with complete data, 
Table 2 Preoperative fine needle aspiration in Group A and Group B, according to 2017 Bethesda System for Reporting Thyroid Cytopathology

\begin{tabular}{|c|c|c|c|c|c|c|c|}
\hline $\begin{array}{l}\text { Cytological } \\
\text { categories }\end{array}$ & \multicolumn{2}{|c|}{ Total } & \multicolumn{2}{|c|}{ Group A } & \multicolumn{2}{|c|}{ Group B } & $P$ value \\
\hline Thyr 1 & 15 & 1.0 & 3 & 0.9 & 12 & 1.1 & 1.000 \\
\hline Thyr 2 & 470 & 32.4 & 152 & 43.6 & 318 & 28.9 & 0.0001 \\
\hline Thyr 3 & 105 & 7.2 & 9 & 2.6 & 96 & 8.7 & 0.0001 \\
\hline Thyr 5 & 75 & 5.2 & 3 & 0.9 & 72 & 6.5 & 0.0001 \\
\hline Thyr 6 & 249 & 17.2 & 5 & 1.4 & 244 & 22.2 & 0.0001 \\
\hline Non eseguito & 280 & 19.3 & 165 & 48.1 & 112 & 10.2 & 0.0001 \\
\hline Total & 1,449 & 100.0 & 349 & 100.0 & 1,100 & 100.0 & \\
\hline
\end{tabular}

Table 3 Postoperative histological exam in Group A and Group B

\begin{tabular}{|c|c|c|c|c|c|c|c|}
\hline Definitive histology & \multicolumn{2}{|c|}{ Total } & \multicolumn{2}{|c|}{ Group A } & \multicolumn{2}{|c|}{ Group B } & $P$ value \\
\hline Benign pathology & 925 & 64.1 & 302 & 87.3 & 623 & 56.7 & 0.0001 \\
\hline Carcinoma & 466 & 32.3 & 31 & 9.0 & 435 & 39.6 & 0.0001 \\
\hline Microcarcinoma & 53 & 3.7 & 13 & 3.8 & 40 & 3.6 & 0.8711 \\
\hline
\end{tabular}

519 neoplasms were found, of which 53 microcarcinomas $(3.7 \%)$ and 466 carcinomas $(32.3 \%)$, while 925 patients $(64.1 \%)$ did not have neoplastic pathologies. Specifically, among 346 patients in Group A, 13 patients were diagnosed with microcarcinoma $(3.8 \%), 31$ were diagnosed with carcinoma (9.0\%), while the remaining $87.3 \%$ did not have neoplastic pathology. Overall, 44 patients in Group A had developed a thyroid malignancy (13 microcarcinomas and 31 carcinomas), with an overall incidence of $12.8 \%$. Among patients in Group B, microcarcinomas represent 3.6\%, carcinomas $39.6 \%$ and patients without neoplasia $56.7 \%$. Overall, 475 patients in Group B had developed cancer (40 microcarcinomas and 435 carcinomas), with an overall incidence of $43.2 \%$ (Table 3) The different incidence of cancer between hyperfunctioning and non-hyperfunctioning patients was statistically significant $(\mathrm{P}<0.001)$. However, it should be notice that the incidence of microcarcinoma is comparable in the two groups.

Incidence of occasional carcinomas and microcarcinomas in patients who did not undergo preoperative cytological examination was calculated too. Among 280 patients who did not carry out preoperative fine needle aspiration, 166 had a thyroid hyperfunction disease and 114 not. In 166 patients suffering from hyperfunction and not undergoing a preoperative cytological study, microcarcinomas have a frequency of $4.2 \%$ (7 patients) and carcinomas a frequency of $11 \%(6.5 \%)$. Among the 114 patients not subjected to fine needle aspiration and not suffering from hyperfunction, microcarcinomas have a frequency of 3.5\% (4 patients) and occasional carcinomas have a frequency of $11.4 \%$ (13 patients). There was no statistically significant difference in the incidence of occult carcinoma and microcarcinoma in the two groups $(\mathrm{P}>0.05)$.

Subsequently, an assessment of the aggressiveness characteristics of the tumors found in patients with hyperfunction and without hyperfunction was carried out, to understand whether the carcinomas found in the two groups presented similar or different aggressiveness and invasiveness, both local and distant. The characteristics examined were sclerosis, oxyphilia, high cells, perithyroid tissue infiltration, angioinvasion, multifocality and lymph node metastases. Patients selected for these analyses were 
Table 4 Analysis of aggressiveness and invasiveness of thyroid malignancy at postoperative histological exam in Group A and Group B

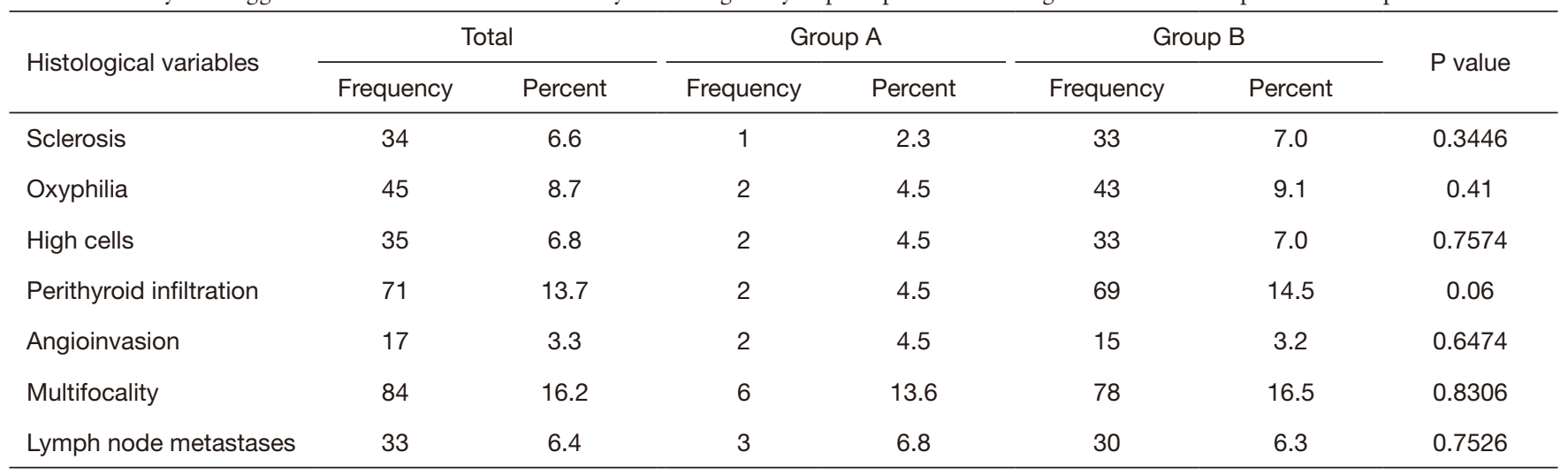

Table 5 Incidence of postoperative complications in Group A and Group B

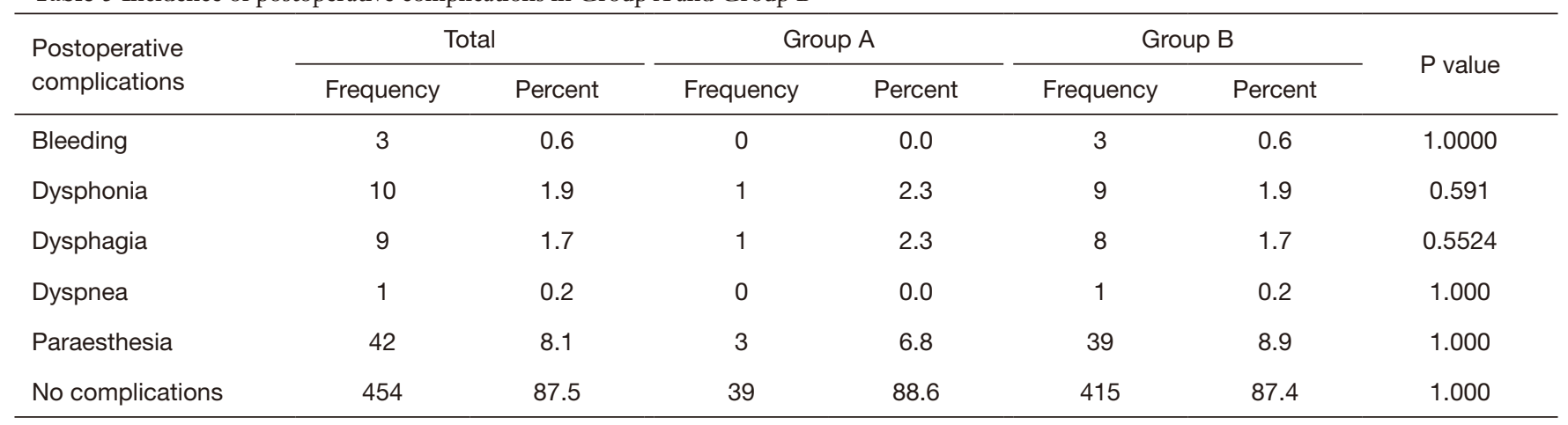

only those with postoperative diagnosis by histological examination of microcarcinoma or carcinoma (519 patients, 53 microcarcinomas and 466 carcinomas). Results are reported in Table 4.

Overall, we did not record a significant difference in the aggressiveness and invasiveness, both local and distant, of malignancy that occur in patients previously suffering from thyroid hyperfunction and those that occur instead in nonhyperthyroid patients $(\mathrm{P}>0.05)$. In fact, the characteristics analyzed occur with overlapping frequency in both Groups $\mathrm{A}$ and $\mathrm{B}$.

Finally, we have considered postoperative complications occurred in both Groups A and B after surgery; such as bleeding, dysphonia, dysphagia, dyspnea, paraesthesia. Only patients with a malignancy at postoperative examination (519 patients) were included in this analysis (Table 5). No statistically significant difference between incidence of postoperative complications in Group A and Group B was revealed $(\mathrm{P}>0.05)$.

\section{Discussion}

Hyperthyroid disease and differentiated thyroid carcinoma are two conditions equally more common in women, with an average age between 53 and 55 years. Patients with hyperthyroidism were mainly subjected to total thyroidectomy, as easily understood, as most of the pathologies that determine this condition are widespread affections of the glandular parenchyma, such as Graves' disease and toxic multinodular goiter. Furthermore, almost half of hyperthyroid patients $(48.1 \%)$ did not undergo preoperative cytological examination, as the presence of a diffuse glandular pathology did not allow identifying any nodular formation worthy of fine needle aspiration. Among hyperthyroid patients subjected to fine needle aspiration, benign disease has the highest prevalence (Thyr $2=43.6 \%$ ), against a prevalence of $28.9 \%$ among nonhyperfunctioning patients. At postoperative diagnosis, the frequency of carcinomas was assessed in patients with prior 
hyperfunction and in patients without hyperthyroidism. In particular, in our study, the incidence of microcarcinomas is similar in the two groups, but the incidence of carcinomas is significantly lower in patients with hyperfunction $(9 \%$ versus $39.6 \%)$.

Considering the results of preoperative cytology, in which hyperthyroid patients are mostly placed in Bethesda's Thyr 2 class, and the results of postoperative histology, hyperthyroidism appears to play a protective role against thyroid neoplasms in the population included in our study, with an overall incidence of neoplasms of $12.8 \%$ in patients with thyroid hyperfunction, of which $9 \%$ carcinomas and $3.8 \%$ microcarcinomas, compared with an incidence of $43.2 \%$, which $39.6 \%$ carcinomas and $3.6 \%$ microcarcinomas, in patients with neoplasia not affected by hyperfunction. Several other studies have reported similar results to ours, describing analogue incidences of malignancy in hyperthyroid patients. In the study by Lin et al. (6) prevalence of thyroid carcinomas in patients with hyperfunction at postoperative diagnosis by histological examination was found of $13.3 \%$. The same study states that the coexistence of thyroid cancer is an eventuality that must always be taken into consideration in the most appropriate therapeutic choice for patients, since an incidence of $13.3 \%$ is lower than the prevalence of carcinomas in nonhyperthyroid patients, but at the same time indicates that the association between hyperthyroidism and carcinomas is not uncommon.

One of the hypothesized explanations of the association between hyperthyroidism and thyroid carcinomas, is the greater use of total thyroidectomy as the treatment of choice in hyperthyroid patients (10), which makes it possible to diagnose a greater number of occult carcinomas. In our study, there was no statistically significant difference in the incidence of occult carcinomas between hyperthyroid and non-hyperthyroid patients. This would also correlate with the finding of the same incidence of microcarcinomas in the two groups of patients. Some other studies have already highlighted that primary hyperthyroidism can be complicated with occult thyroid carcinoma, and this is commonly found in female patients $(11,12)$. Preoperative diagnosis is difficult and ultrasound is still the major examining method, while frozen section can increase the detection rate. However, the incidence of occult neoplasms in patients not subjected to total fine needle aspiration (both hyperthyroid and non-hyperthyroid) is significantly lower than the incidence of occult neoplasms in patients who have undergone preoperative cytological examination. This result is attributable to the more frequent association of a thyroid neoplasm with concomitant thyroid nodular pathologies rather than to diffuse hyperplasia or hyperfunctions of the gland.

In this regard, a recent study considered hyperthyroid patients undergone to surgery and revealed a higher than expected rate of malignancy at pathological exam. Moreover, those with hyperfunctional nodular disease had both the highest rates of thyroid malignancy and histological tumor aggressiveness. Patients with both hyperfunctioning disease and carcinoma presented a toxic adenoma in $50 \%$, toxic MNG in 24\% and Graves' disease in $16 \%$. Therefore, they suggested that hyperthyroid patients with nodularity on ultrasound examination should perform appropriate FNA assessment to value the risk of malignancy (13).

Several studies confirm that the association between hyperthyroidism and thyroid carcinomas is low but not rare (14), although with a lower frequency than in the general nonhyperthyroid population, identifying hyperthyroidism as a protective condition against neoplasms, but still recommend the execution of a cytological examination in the case of a nodular pathology in a hyperthyroid patient before performing a treatment with radioiodine or surgical treatment $(2,15,16)$. Some authors have also hypothesized that neoplasms associated with hyperthyroidism, whether papillary or follicular, could be more aggressive, since very often, there are lymph node metastases, distant metastases and the de-differentiation of the neoplasm in an undifferentiated carcinoma $(17,18)$.

TSH acts as a promoter for the proliferation of normal and neoplastic thyroid cells. Although suppressed in hyperthyroid patients, many studies point out that thyroidstimulating antibodies (Trab) present in Basedow Graves' disease can induce tumor proliferation by activating TSH receptors (19-21). In this respect, it is important to note that ours is an iodine-deficient region, therefore the consequent subclinical hypothyroidism causes a chronic increase in serum TSH levels, and this results in the high incidence of thyroid carcinoma even in euthyroid patients. Also an Italian study from Medas et al., working in an analogue iodine-deficiency area, described high tumor incidences both in hyperthyroid patients and not $(20.6 \%$ versus $34.3 \%$ ), similar to ours (21). According with Trabstimulating hypothesis, Filetti et al. had observed a higher finding of thyroid carcinoma in Grave's disease than in toxic solitary nodule. However, patients with Graves' disease presented more frequently aggressive malignancy while these were uncommon in patients with toxic adenoma. 
Based on these results, we can hypothesize that TSH and thyroid stimulating antibodies play a fundamental role in the genesis, growth and metastatic spread of thyroid cancer. Indeed, tumors with aggressive behavior are less frequent in patients with low or suppressed serum TSH levels and, on the other hand, more frequent in those with chronic stimulation of the TSH receptor by thyroid stimulating antibodies (19).

However, in most cases, hyperthyroid malignancies reported are small, less than $1 \mathrm{~cm}$, and have an excellent prognosis (22). Alvarez et al. reported that $60 \%$ of patients undergone to thyroidectomy for hyperthyroidism had tumor with a diameter $<1 \mathrm{~cm}$, and overall, all malignancy were $<3 \mathrm{~cm}$ in size. The clinical impact of these small cancers is still unclear, especially after the introduction of the 2015 ATA guidelines in which tumor dimensions influence the extent of surgery (13).

To evaluate aggressiveness and invasiveness, both local and distant, of the tumors occurred in patients included in the study, we examined some histological characteristics, including sclerosis, oxyphilia, high cells, local infiltration, angioinvasion, multifocality and lymph node metastases. Our study showed that all these characteristics occur with comparable frequencies in both groups of patients analyzed. It can therefore be affirmed not only that hyperthyroidism is a protective condition against thyroid carcinoma but also that in hyperthyroid patients who develop a carcinoma the latter does not present with greater severity, aggressiveness and invasiveness, but also comparable in every respect to carcinomas diagnosed in non-hyperthyroid patients. Medas et al. reported a higher incidence of node metastasis and a worst prognosis in hyperthyroid patients at 5-year follow up. Unfortunately, our study is lack of this prospective evaluation (21).

Finally, we have evaluated the incidence of postoperative complications after thyroidectomy for malignancy in hyperfunctioning and non-hyperfunctioning patients. Many articles in literature report an increased incidence of bleeding (23), transient hypocalcemia (24-26) and even a higher risk of vocal-cord paralysis and tracheostomy in hyperfunctioning patients (27). In spite of these reports, we did not find statistically significant differences in the incidences of bleeding, dysphonia, dysphagia, dyspnea and paresthesia in our series. In our opinion, the procedures performed in the two groups of patients have the same safety and we had priory obtained the same results considering the impact of hyperfunction even in minimally invasive procedures (28).

\section{Conclusions}

Our study reveals that in patients suffering from hyperfunctioning thyroid disease, undergoing thyroid surgery, the incidence of neoplasia on definitive histological examination is lower. Furthermore, the carcinomas found in patients with thyroid hyperfunction do not show greater aggressiveness or invasiveness, both local and distant, but their characteristics are completely comparable with those of the carcinomas found in patients who are not affected by thyroid hyperfunction. However, hyperfunctioning and non-hyperfunctioning patients presented an equal incidence of microcarcinomas and occult carcinomas on the postoperative histological examination so we can no longer consider hyperthyroidism a protective condition against the onset of differentiated thyroid carcinoma. Therefore, in patients with hyperthyroidism, it is of fundamental importance to carry out a preoperative cytological examination to plan a correct therapeutic program.

\section{Acknowledgments}

Funding: None.

\section{Footnote}

Reporting Checklist: The authors have completed the STROBE reporting checklist. Available at http://dx.doi. org $/ 10.21037 /$ gs-20-688

Data Sharing Statement: Available at http://dx.doi. org/10.21037/gs-20-688

Conflicts of Interest: All authors have completed the ICMJE uniform disclosure form (available at http://dx.doi. org/10.21037/gs-20-688). The authors have no conflicts of interest to declare.

Etbical Statement: The authors are accountable for all aspects of the work in ensuring that questions related to the accuracy or integrity of any part of the work are appropriately investigated and resolved. The study was conducted in accordance with the Declaration of Helsinki (as revised in 2013). The study was approved by institutional ethics board of Parma - AVEN (No. 1009/2019/OSS*/ AOUPR) and individual consent for this retrospective analysis was waived. 
Open Access Statement: This is an Open Access article distributed in accordance with the Creative Commons Attribution-NonCommercial-NoDerivs 4.0 International License (CC BY-NC-ND 4.0), which permits the noncommercial replication and distribution of the article with the strict proviso that no changes or edits are made and the original work is properly cited (including links to both the formal publication through the relevant DOI and the license). See: https://creativecommons.org/licenses/by-nc-nd/4.0/.

\section{References}

1. Farbota LM, Calandra DB, Lawrence AM, et al. Thyroid carcinoma in Graves' disease. Surgery 1985:98:1148-53.

2. Gabriele R, Letizia C, Borghese $M$, et al. Thyroid cancer in patients with hyperthyroidism. Horm Res 2003;60:79-83.

3. Gerenova J, Buysschaert M, de Burbure CY, et al. Prevalence of thyroid cancer in Graves' disease: a retrospective study of a cohort of 103 patients treated surgically. Eur J Intern Med 2003;14:321-5.

4. Ergin AB, Saralaya S, Olansky L. Incidental papillary thyroid carcinoma: clinical characteristics and prognostic factors among patients with Graves' disease and euthyroid goiter, Cleveland Clinic experience. Am J Otolaryngol 2014;35:784-90.

5. Staniforth JUL, Erdirimanne S, Eslick GD. Thyroid carcinoma in Graves' disease: A meta-analysis. Int J Surg 2016;27:118-25.

6. Lin CH, Chiang FY, Wang LF. Prevalence of thyroid cancer in hyperthyroidism treated by surgery. Kaohsiung J Med Sci 2003;19:379-84.

7. Hales IB, McElduff A, Crummer P, et al. Does Graves' disease or thyrotoxicosis affect the prognosis of thyroid cancer. J Clin Endocrinol Metab 1992;75:886-9.

8. Delange F, Lecomte P. Iodine supplementation: benefits out-weigh risks. Drug Saf 2000;22:89-95.

9. Cibas ES, Ali SZ. The 2017 Bethesda System for Reporting Thyroid Cytopathology. Thyroid 2017;27:1341-6.

10. Ruggieri M, Scocchera F, Genderini M, et al. Hyperthyroidism and concurrent thyroid carcinoma. Eur Rev Med Pharmacol Sci 1999;3:265-8.

11. Wu X, Yu JC, Kang WM, et al. Surgical Diagnosis and Treatment of Primary Hyperthyroidism Complicated with Occult Thyroid Carcinoma. Zhongguo Yi Xue Ke Xue Yuan Xue Bao 2015;37:402-5.

12. More $Y$, Khalil AB, Mustafa H, et al. Incidental Thyroid cancer in patients undergoing surgery for hyperthyroidism. Am J Otolaryngol 2020;41:102187.
13. Alvarez AL, Mulder M, Handelsman RS, et al. High Rates of Underlying Thyroid Cancer in Patients Undergoing Thyroidectomy for Hyperthyroidism. J Surg Res 2020;245:523-8.

14. Tam AA, Ozdemir D, Alkan A, et al. Toxic nodular goiter and thyroid cancer: Is hyperthyroidism protective against thyroid cancer? Surgery 2019;166:356-61.

15. Gul K, Di Ri Koc A, Ki Yak G, et al. Thyroid carcinoma risk in patients with hyperthyroidism and role of preoperative cytology in diagnosis. Minerva Endocrinol 2009;34:281-8.

16. Daou R. Hyperthyroïdie et cancer de la thyroïde Hyperthyroidism and cancer of the thyroid. Chirurgie 1998;123:604-8.

17. Ozaki O, Ito K, Kobayashi K, et al. Thyroid carcinoma in Graves' disease. World J Surg 1990;14:437-40; discussion 440-1.

18. Pazaitou-Panayiotou K, Perros P, Boudina M, et al. Mortality from thyroid cancer in patients with hyperthyroidism: the Theagenion Cancer Hospital experience. Eur J Endocrinol 2008;159:799-803.

19. Filetti S, Belfiore A, Amir SM, et al. The role of thyroidstimulating antibodies of graves' disease in differentiated thyroid cancer. N Engl J Med 1988;318:753-9.

20. Mazzaferri EL. Thyroid cancer and Graves' disease. J Clin Endocrinol Metab 1990;70:826-9.

21. Medas F, Erdas E, Canu GL, et al. Does hyperthyroidism worsen prognosis of thyroid carcinoma? A retrospective analysis on 2820 consecutive thyroidectomies. J Otolaryngol Head Neck Surg 2018;47:6.

22. Vini L, Hyer S, Pratt B, et al. Good prognosis inthyroid cancer found incidentally at surgery for thyrotoxicosis. Postgrad Med J 1999;75:169-70.

23. Liu J, Sun W, Dong W, et al. Risk factors for postthyroidectomy haemorrhage: a meta-analysis. Eur J Endocrinol 2017;176:591-602.

24. Edafe O, Antakia R, Laskar N, et al. Systematic review and meta-analysis of predictors of post-thyroidectomy hypocalcaemia. Br J Surg 2014;101:307-20.

25. Hallgrimsson P, Nordenström E, Bergenfelz A, et al. Hypocalcaemia after total thyroidectomy for Graves' disease and for benign atoxic multinodular goitre. Langenbecks Arch Surg 2012;397:1133-7.

26. Welch KC, McHenry CR. Total thyroidectomy: is morbidity higher for Graves' disease than nontoxic goiter? J Surg Res 2011;170:96-9.

27. Rubio GA, Koru-Sengul T, Vaghaiwalla TM, et al. Postoperative Outcomes in Graves' Disease Patients: 
Results from the Nationwide Inpatient Sample Database. Thyroid 2017;27:825-31.

28. Del Rio P, Cozzani F, Puteo N, et al. IONM and

Cite this article as: Bonati E, Bettoni S, Loderer T, Del Rio P. Can we still consider thyroid hyperfunction a protective condition for the onset of thyroid cancer? Gland Surg 2021;10(4):1359-1367. doi: 10.21037/gs-20-688 thyroidectomy in benign thyroid disease. Analysis of adverse events. G Chir 2019;40:174-81. 